\title{
Therapeutic potential of oleic acid nanovesicles prepared from petroleum ether extract of Sargassum binderi in streptozotocin-induced diabetic wound in Wistar rats
}

\author{
Sivakumar Sivagurunathan Moni ${ }^{1 *}$, Mohammad Firoz Alam², Hafiz A Makeen ${ }^{3}$, \\ Aamena Jabeen ${ }^{1}$, Syeda Sanobar ${ }^{4}$, Rahimullah Siddiqui ${ }^{2}$, Remesh Moochikkal ${ }^{5}$, \\ Soliman Fouda ${ }^{1}$ \\ ${ }^{1}$ Divison of Immunology and Pharmaceutical Biotechnology, Department of Pharmaceutics, Faculty of Pharmacy, ${ }^{2}$ Department \\ of Pharmacology and Toxicology, Faculty of Pharmacy, ${ }^{3}$ Pharmacy Practice Research Unit (PPRU), Clinical Pharmacy \\ Department, Faculty of Pharmacy, ${ }^{4}$ Department of Pharmacognosy, Faculty of Pharmacy, ${ }^{5}$ Department of Biology, Faculty of \\ Science, Jazan University, Jazan, Saudi Arabia
}

*For correspondence: Email: drsmsivakumar@gmail.com

\begin{abstract}
Purpose: To study the effectiveness of phyto-oleic acid nanovesicles (PONVs) developed from Sargassum binderi (an alga) in healing diabetic wound in a rat model, and to establish the associated changes in cytokine network.

Methods: Phyto-extract was obtained from the whole plant of Sargassum binderi by Soxhlet extraction using petroleum ether as solvent. The crude extract was subjected to phytochemical analysis and used in the formulation of POVNs. The PONVs were formulated by entrapping petroleum ether extract of Sargassum binderi using the film hydration technique. Wound healing property was determined by measuring both pro-inflammatory and anti-inflammatory cytokines using enzyme-linked immunosorbent assay (ELISA).

Results: Tannins and steroids were the major components of the petroleum ether extract of Sargassum binderi. Serum cytokine levels were increased after inducing diabetes and creating the wound. The serum levels of IL-2, TNF- $\alpha$ and IL-1 $\beta$ were $37.3 \pm 3.3,76.3 \pm 5.2$ and $3307.6 \pm 350 \mathrm{pg} / \mathrm{ml}$, respectively. Treatment with PONVs modulated the serum cytokine levels through significant decreases in serum IL2, TNF- $\alpha, I L-1 \beta$ levels, and significant elevation of serum IL-4.

Conclusion: These results indicate that PONVs have promising potentials for application as topical treatment for diabetic wounds.
\end{abstract}

Keywords: Brown algae, Sargassum binderi, Oleic acid nanovesicles, Diabetic wound, Cytokines

\begin{abstract}
This is an Open Access article that uses a funding model which does not charge readers or their institutions for access and distributed under the terms of the Creative Commons Attribution License (http://creativecommons.org/licenses/by/4.0) and the Budapest Open Access Initiative (http://www.budapestopenaccessinitiative.org/read), which permit unrestricted use, distribution, and reproduction in any medium, provided the original work is properly credited.
\end{abstract}

Tropical Journal of Pharmaceutical Research is indexed by Science Citation Index (SciSearch), Scopus, International Pharmaceutical Abstract, Chemical Abstracts, Embase, Index Copernicus, EBSCO, African Index Medicus, JournalSeek, Journal Citation Reports/Science Edition, Directory of Open Access Journals (DOAJ), African Journal Online, Bioline International, Open-J-Gate and Pharmacy Abstracts

\section{INTRODUCTION}

Wound healing is a series of complex processes characterized by various phases such as inflammation, epithelialization, angiogenesis, matrix deposition, and remodelling [1]. Wounds in diabetes mellitus have significant complications that are highly linked to 
neuropathy [2]. Progression in the healing of diabetic wounds is associated with decreased keratinocytes, fibroblast migration of immune cells into the endothelial cells, and angiogenesis [3-5]. Cytokines are small molecular weight proteins that can reach the wound cells through autocrine, paracrine, and endocrine fashions, which are crucial factors in the inflammatory response. Specifically, cytokines have prominent roles in the wound healing process, ranging from pro-inflammatory to anti-inflammatory responses. The present state of treatment for diabetic wounds still faces significant challenges such as poor entry of drugs into the wound cells, and development of drug resistance.

Research efforts have resulted in the development of newer and wider-spectrum antibiotics. However, there is no assurance that wound-associated organisms will not develop resistance to these newer antibiotics [6]. Moreover, although the use of drugs of herbal origins as alternatives for the development of antibacterial agents has almost reached its maximum potential, their effectiveness is debatable till date. Consequently, researchers on drug discovery have shifted their attention to seaweeds for the past three decades, with the aim of developing antibacterial agents to replace the present antibiotic treatment regimen. In this respect, biomolecules with antibacterial properties and modulating effects on cytokines are highly significant in therapeutic management options for diabetic wounds.

The potential of Sargassum binderi which is available in the Red Sea of Jazan, Saudi Arabia, has not yet been explored as a pharmaceutical agent. The antibacterial effects of petroleum ether extract and oleic acid vesicles of Sargassum binderi have been demonstrated by Sivakumar et al [7]. In the present study, the immuno-regulatory effect of oleic acid vesicles prepared by entrapping petroleum ether extract of Sargassum binderi was investigated. In addition, the wound-healing effect of the oleic acid vesicles was studied in streptozotocin (STZ)-induced diabetic wounds in Wistar rats to ascertain their potential as topical application for treating diabetic wounds.

\section{EXPERIMENTAL}

\section{Collection and identification of seaweed}

Sargassum binderi was collected from the Red Sea, Al Murjan beach, Jazan, Saudi Arabia, and washed thoroughly using fresh water to remove extraneous substances and epiphyte adherents on the samples. Sargassum binderi Sonder ex J.
Agardh was identified by Dr. Remesh Moochikkal, herbarium curator, Herbarium of Jazan University, Department of Biology, Jazan University, Jazan, Saudi Arabia. A voucher specimen with ref no. 1210(JAZUH) was deposited at the institution's herbarium for future reference.

\section{Phytochemical extraction}

The seaweed was subjected to air-drying under shade for 15 days, after which the sample was sliced into small pieces and powdered using a grinder. The coarsely-powdered samples were pooled and kept in an airtight container. The powdered sample was extracted in a Soxhlet apparatus using hot, continuous percolation with petroleum ether. In essence, $200 \mathrm{~g}$ of dried Sargassum binderi powder was placed in a Soxhlet apparatus and extracted with petroleum ether as a solvent. The total ensemble was set up on a heating mantle. The solvent was heated to about $60^{\circ} \mathrm{C}$, and the solvent extraction was performed continuously for $4 \mathrm{~h}$. Several $200-\mathrm{g}$ batches of powder were similarly extracted. After extraction, the extracts were transferred into separate glass beakers which were kept open to permit solvent evaporation and air-drying of the extract. The dry samples were collected by scraping them off from the glass beaker, and were pooled and weighed. The pooled sample was then subjected to phytochemical analysis, and its PONV's formulations for diabetic wound healing.

\section{Preparation of oleic acid vesicles}

The PONV was prepared by using the film hydration technique. The extract and oleic acid were mixed in a 1:1 proportion. The mixture was sonicated for about $5 \mathrm{~min}$ and then mixed with methanol in $1: 1$ proportion. The mixture was heated on a heating mantle at $50{ }^{\circ} \mathrm{C}$ for $60 \mathrm{~min}$ to form a thin film following solvent removal. Finally, the PONV was eluted in methanol and the resultant vesicles were used for in vivo studies on wound healing properties.

\section{In vivo wound healing property}

Healthy male Wistar rats weighing $170-200 \mathrm{~g}$ were used. They were acclimatized to laboratory conditions and handled in strict compliance with the guidelines of the International standard and Institutional Animal Care and Use committee (IACUC) [8]. The rats were assigned randomly to three different groups (6 rats/group) and categorized as follows:

Trop J Pharm Res, November 2018; 17(11): 2124 
Group 1: Normal control group: The animals did not receive streptozotocin (STZ) or PONVs.

Group 2: Disease control group (diabetes induction and diabetic wound): The rats received intraperitoneal injection of STZ (at a dose of 50 $\mathrm{mg} / \mathrm{kg}$ body weight dissolved in $0.9 \% \mathrm{w} / \mathrm{v}$ of sodium chloride (normal saline). The wound was created according to the procedure established by Mendes et al [9]. In this process, the rats were anesthetized with intraperitoneal injection of xylazine hydrochloride $(10 \mathrm{mg} / \mathrm{kg})$ and ketamine hydrochloride $(25 \mathrm{mg} / \mathrm{kg})$. Then, the dorsal surface hair was trimmed with an electric shaver, followed by hair removal using commercially available hair remover. An area of skin was cleaned with $10 \%$ povidone iodine solution, and a 6-mm diameter skin was removed with a sterile surgical blade to create a circular wound on the upper back of the rat (Figures 2 A \& B).

Group 3: Treatment group: The rats received intraperitoneal injection of STZ $(50 \mathrm{mg} / \mathrm{kg}$ body weight) dissolved in normal saline, and diabetic wound as in group 2. In addition, they were treated with $500 \mu \mathrm{l}$ of PONVs (twice daily) by spreading them on the wound for seven days. After induction of diabetes, blood glucose levels were measured at predetermined time intervals in the morning and evening. The level of glucose was measured on the tail-vein blood using a standard commercially available glucometer.

The blood glucose level was checked and the diabetes was confirmed by the elevation of fasting blood glucose above $250 \mathrm{mg} / \mathrm{dL}$. On day 6 , fasting blood samples were collected and allowed to clot. The serum samples obtained by centrifugation were kept in a refrigerator at $2-8$ ${ }^{\circ} \mathrm{C}$ prior for use in the assay of cytokine levels. The pro-inflammatory cytokines, IL-1 $\beta$, IL-2), IL4 , and TNF- $\alpha$ levels were estimated as follows.

\section{Serum IL-1 $\beta$}

The level of IL- $1 \beta$ in serum was measured quantitatively by using IL- $1 \beta$ rat ELISA kits (ABCAM, USA). The assay employs simple step sandwich ELISA to determine the IL-1 $\beta$ level in the serum sample. Simultaneously, standards and samples were pipetted into the respective wells and incubated at room temperature for 2.5 $\mathrm{h}$ after closing the ELISA plate with a lid. During incubation, the IL-1 $\beta$ present in the sample was bound to the wells that had been coated with the immobilized specific antibody. After incubation, the wells were washed thoroughly with $1 \mathrm{X}$ washing solution using Biotek ELISA washer (Elx50, USA). Then, $1 \mathrm{X}$ biotinylated anti-rat IL-1 beta antibody was added to each well. The plate was incubated for $1 \mathrm{~h}$ at room temperature with mild shaking.

After incubation, the plate was washed as previously described, and the unbound biotinylated antibody was removed. Then, horseradish peroxidase (HRP)-conjugated streptavidin was added to the wells, and the wells were again washed as described in the previous step. A substrate solution, tetramethylbenzidine (TMB) was added to the wells and incubated for $30 \mathrm{~min}$ at room temperature in a dark with mild shaking. This was followed by addition of the stopping solution. The thickness of the developed colour which is proportional to the amount of IL-1, was measured at $450 \mathrm{~nm}$ using a Biotek ELISA reader (ELX 800, USA). The absorbance was an indication of the amount of IL-1 beta bound to the specific antibody. The sample concentration was calculated by extrapolation from a standard curve.

\section{Serum IL-2}

The level of IL-2 in serum was estimated quantitatively using IL-2 rat ELISA kit, ABCAM, USA. Sandwich ELISA was used to determine the IL-2 level in serum samples. Standards and samples were pipetted simultaneously and dispensed into the respective wells. The plate was closed with a lid and incubated at $4{ }^{\circ} \mathrm{C}$ overnight with mild shaking. Thereafter, the solution was discarded and the wells were washed with $1 \mathrm{X}$ washing solution using Biotek ELISA washer ELX 50, USA. Then, 1X biotinylated anti-rat IL-2 antibody was added and incubated at room temperature for $1 \mathrm{~h}$ with mild shaking. The plate was washed as previously described, and the unbound biotinylated antibody was removed. Then, HRP-conjugated streptavidin was added to the wells, followed by incubation for $45 \mathrm{~min}$ at room temperature.

The wells were again washed as described in the previous step. Finally, TMB solution was added to all the wells, followed by incubation in the dark with mild shaking at room temperature for 30 $\mathrm{min}$. Thereafter, the stopping solution was added and the concentration of IL-2 was measured by reading absorbance at $450 \mathrm{~nm}$ using a ELISA reader (Biotek ELX 800). The concentration of IL2 was obtained by extrapolation from a standard curve.

\section{Serum IL- 4}

Serum IL-4 was quantitatively estimated in a sandwich ELISA assay using IL-4 rat ELISA kits (ABCAM, USA). The procedure was similar to that used for IL -2 estimation, except that $1 \mathrm{X}$ 
biotinylated anti-rat IL-4 antibody was added. Absorbance of the colour developed was read at $450 \mathrm{~nm}$ using a ELISA reader (Biotek ELX 800). The concentration of TNF- $\alpha$ was obtained by extrapolation from a standard curve.

\section{Serum TNF- $\alpha$}

Serum TNF-a level was quantitatively estimated using a simple step sandwich ELISA assay. Samples and standard were simultaneously pipetted into the respective wells. Then, 1X biotinylated anti-TNF- $\alpha$ was put in the wells and the plate was incubated for $3 \mathrm{~h}$ at room temperature. Thereafter, the plate was rinsed thrice with $1 \mathrm{X}$ washing Buffer using Biotek ELISA washer (ELX50, USA), followed by addition of $1 X$ streptavidin-HRP solution to the wells. The plate was closed and incubated at room temperature for $30 \mathrm{~min}$, after which it was rinsed with washing buffer as described earlier. Then, TMB substrate solution was added to all the wells, followed by incubation in the dark at room temperature for 20 $\min$. The reaction was stopped by addition of the stopping solution, and the absorbance of the resultant color was read at $450 \mathrm{~nm}$ using an ELISA reader (Biotek ELX 800). The concentration of TNF- $\alpha$ was extrapolated from a standard curve.

\section{Statistical analysis}

The data are presented as mean \pm standard deviation SD). The statistical significance of differences between treatment groups were determined using one-way analysis of variance (ANOVA) and Dunnet multiple comparison test. All analyses were performed with GraphPad InStat 3.10 software, USA. The level of significance was set at $p<0.001$.

\section{RESULTS}

Results from hot continuous percolation method showed that the petroleum ether extract of Sargassum binderi predominantly contained tannins and steroids. The PONVs were successfully formulated. The in vivo wound healing study revealed that PONVs possess significant wound-healing properties. The results shown in Table1 indicate that group 2 rats treated with STZ exhibited very high and significant increases in the serum levels of IL-1 $\beta$, IL-2, and TNF- $\alpha(p<0.001)$.

However, in the treatment group (group 3), exposure to PONVs successfully reversed these increases by significant decreases in serum levels of IL-1 $1 \beta$, IL-2, and TNF- $\alpha$. The decreases in these pro-inflammatory cytokines were consistent with the wound-healing results of PONVs as depicted in Table 1. Interestingly, PONVs treatment led to significant increases in the serum level of the anti-inflammatory cytokine IL- $4(p<0.001)$, when compared with the other groups (Table 1). The results in Figure 1 demonstrate stepwise, the in vivo wound-healing process of PONV as self-exemplary.
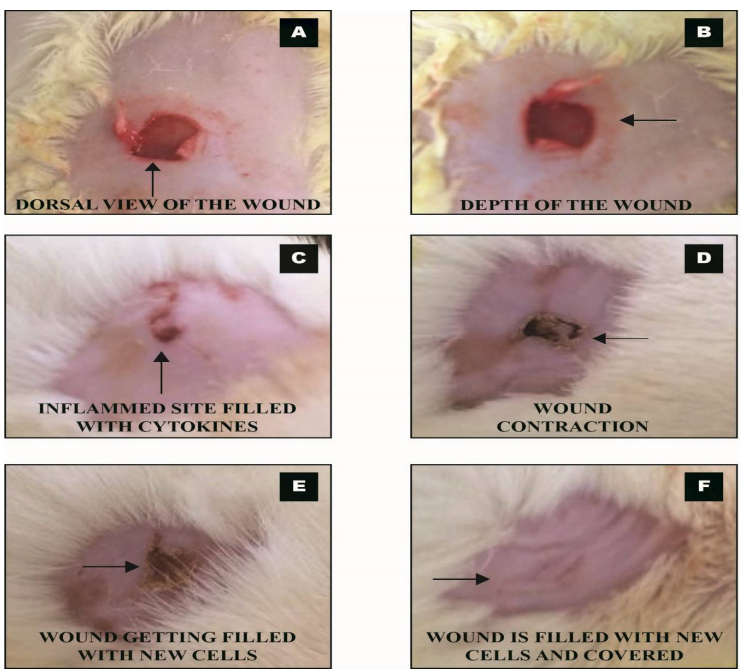

Figure 1: Wound healing property of PONV in various stages of healing processes of diabetic wound. Fig1 (A\&B) represents the diabetic wound created in rats; Fig (C to F) represents the wound healing processes

Table 1: Pro-inflammatory and anti-inflammatory cytokine levels before and after treatment of PONV in various treatment group

\begin{tabular}{|c|c|c|c|c|c|c|}
\hline \multirow[b]{2}{*}{$\begin{array}{l}\text { Cytokine } \\
\text { level } \\
(\mathrm{pg} / \mathrm{ml})\end{array}$} & \multicolumn{6}{|c|}{ Treatment groups } \\
\hline & Group 1 & Group 2 & $\begin{array}{c}\text { Fold } \\
\text { increase } \\
\text { (conc.) }\end{array}$ & Group 3 & $\begin{array}{c}\text { Fold increase/ } \\
\text { decrease } \\
\text { (conc.) }\end{array}$ & $P$-value \\
\hline IL-2 & $1.48 \pm 0.5$ & $37.3 \pm 3.3$ & 25.2 & $1.688 \pm 0.4^{\star *}$ & $22.9^{*}$ & $<0.001$ \\
\hline TNF- $\alpha$ & $2.49 \pm 0.61$ & $76.3 \pm 5.2$ & 30.64 & $3.25 \pm 0.65^{\star *}$ & $23.56^{*}$ & $<0.001$ \\
\hline IL-1 $\beta$ & $106.3 \pm 6.8$ & $3307.6 \pm 350$ & 31.1 & $137.7 \pm 23.3^{\star *}$ & $24.02^{*}$ & $<0.001$ \\
\hline IL-4 & $1.67 \pm 0.28$ & $27 \pm 3.63$ & 16.16 & $38.35 \pm 5.34^{* *}$ & $22.96^{\#}$ & $<0.001$ \\
\hline
\end{tabular}

Each value is e mean \pm SD $(n=6) ; " p<0.001$ (99.9\% confidence interval) is very extremely significant when compared to normal group. *Fold decreased in concentration $(\mathrm{pg} / \mathrm{ml})$ after treatment' \# Fold increased in concentration $(\mathrm{pg} / \mathrm{ml})$ after treatment 


\section{DISCUSSION}

The present study focused on establishing the effect of PONVs on wound healing in diabetic rats by modulating pro-inflammatory cytokine levels. Streptozotocin (STZ) is a potent chemical popularly used for the induction of diabetes mellitus. It produces a toxic effect by destructing the $\beta$ cells of the islet of Langerhans $[10,11]$. In this study, STZ-treated rats significantly developed diabetes mellitus. It is known that IL$1 \beta$ is a predominant cytokine present in inflammatory conditions linked to diabetic mellitus [12].

Pro-IL-1 $\beta$ is an inactive precursor protein that is converted by the enzyme caspase 1 to active IL$1 \beta$ which is associated with defective secretion of insulin as well the development of insulin resistance [13-15]. In the present study, serum IL-1 $\beta$ level was upregulated expressively in the STZ-injected rats, when compared to the normal control group. However, treatment with PONV brought about significant reduction in serum IL$1 \beta$ level. Generally, T Helper 2 (CD4+) cells predominantly produce a low molecular weight protein called interleukin-2 (IL-2). Cytotoxic T (CD8+), natural killer (Nk), and natural killer T (Nk T) cells are also involved to a lesser extent in the production of IL-2 which has proinflammatory and anti-inflammatory effector functions.

It has been shown that IL-2 is a messenger that provides signals to various lymphocytes during proliferation and differentiation, leading to immune responses and maintenance of homeostasis [11]. The present study showed that the IL-2 level increased in the group 2 animals that received STZ but the increase was reversed significantly after treatment with PONV in group 3 animals. It has been suggested that IL-2 signals affect the action of CD8+ cells while mounting the immune response and therefore, the inflammatory response action is ineffective [16]. In contrast, the present study showed the predominant inflammatory effect of IL-2 which was significantly reduced by treatment with PONV.

The cytokine TNF- $\alpha$, a low molecular weight protein derived mainly from macrophages, is involved in inflammatory mechanism and closely associated with insulin resistance [17]. It is an inflammatory biomarker that plays a role in regulating the inflammatory processes in diabetes [18]. Consistent with the earlier report, the present study showed that STZ-induced diabetes was accompanied with significant increases in serum TNF- $\alpha$ level. The results indicate that the STZ treatment induces insulin resistance. It is very interesting to note that after treatment with PONVs, the reduction in serum TNF- $\alpha$ level and the attendant reduction in inflammation occurred with healing of the diabetic wound. Interleukin-4 (IL-4) plays an important role in regulating the anti-inflammatory responses and growth factor for both $\mathrm{B}$ and $\mathrm{T}$ lymphocytes.

It has been reported that the production of IL-4 is very high in diabetes mellitus accompanied with chronic periodontal disease [19]. Interestingly, the present study has demonstrated that IL-4 level was very high after the treatment with PONVs, when compared to the untreated STZinduced diabetic group. Thus, PONVs are potent immune boosters which modulate the inflammatory response and heal wounds. In a previous study, it was reported that IL-4 inhibited the secretion of IL- $1 \beta$ and TNF- $\alpha$ using activated monocytes [20]. Similarly, the present study showed that after treatment with PONVs, serum IL-4 level increased while serum IL-1 $\beta$, IL-2, and TNF- $\alpha$ levels decreased. It has been reported that IL-2 plays an important role in Th2 cell differentiation that stabilizes the accessibility of the IL-4 gene and efficiently primes IL-4 production [21,22].

\section{CONCLUSION}

The results obtained in the present study indicate that the phyto-extract of Sargassum binderi entrapped in oleic acid nanovesicles (PONVs) possesses effective in vivo wound-healing property in a diabetic rat wound model. The wound-healing occurred via a mechanism involving downregulation of pro-inflammatory cytokines and upregulation of anti-inflammatory cytokines.

\section{DECLARATIONS}

\section{Acknowledgement}

The authors acknowledge the Deanship of Scientific Research, Jazan University for funding the project (reference no. 37/7/000102).

\section{Conflict of Interest}

No conflict of interest associated with this work.

\section{Contribution of Authors}

The authors declare that this work was done by the authors named in this article and all liabilities 
pertaining to claims relating to the content of this article will be borne by them.

\section{REFERENCES}

1. Cory EDC, Laurie PS. The cytokine milieu of diabetic wounds. Diabetes Manag 2015; 5(6): 525-537.

2. Kleopatra A, John D. Management of diabetic foot ulcers. Diabetes Ther 2012; 3(4): 1-15.

3. Qing $C$. The molecular biology in wound healing \& nonhealing wound. Chin J Traumatol 2017; 20(4): 189-193.

4. Brem H, Tomic CM. Cellular and molecular basis of wound healing in diabetes. J Clin Invest 2007; 117(5): 1219 - 1222.

5. Liu ZJ, Omaida CV. Hyperoxia, endothelial progenitor cell mobilization, and diabetic wound healing. Antioxid Redox Signal 2008; 10(11): 1869-1882.

6. Sivakumar SM, Safhi MM, Aamena J, Foziyah Z, Farah I, Bagul US, Sanobar S, Tarique A, Rahimullah $S$, Mohammad Abdul Hakeem $S$ et al. Therapeutic potential of chitosan nanoparticles as antibiotic delivery system: challenges to treat multiple drug resistance. Asian J Pharm 2016; 9(6): S1-S6.

7. Sivakumar SM, Alam MF, Safhi MM, Aamena J, Syeda S. Targeting to develop novel anti-bacterial drug from seaweeds of Jazan province by fractionation, phytochemical analysis and in vitro anti-bacterial evaluation. 7th Research Project, reference No. 37/7/000102, Deanship of Scientific Research, Jazan University, Jazan, Kingdom of Saudi Arabia, Nov 2016Nov 2017.

8. National, Research Council, Guide for the Care and Use of Laboratory Animals, 7th ed. National Academy Press, Washington DC, 1996.

9. João JM, Clara IL, Dolores PB, Andreia LP. A Rat Model of Diabetic Wound Infection for the Evaluation of Topical Antimicrobial Therapies. Comp Med 2012; 62(1): 37-48.

10. Srinivasan K, Ramarao P. Animal models in type 2 diabetes research: an overview. Indian J Med Res 2007; 125: 451-472.

11. Ghosh S, Bhattacharyya S, Rashid K, Parames CS. Curcumin protects rat liver from streptozotocin induced diabetic pathophysiology by counteracting reactive oxygen species and inhibiting the activation of p53 and MAPKs mediated stress response pathways. Toxicol Rep 2015; 2: 365-376.
12. Böni-Schnetzler $M$, Donath $M Y$. Increased IL-1 activation, the culprit not only for defective insulin secretion but also for insulin resistance? Cell Res 2011; 21(7): 995-997.

13. Stienstra $R$, Joosten $L A$, Koenen $T$, van Tits $B$, van Diepen JA, van den Berg SA, Rensen PC, Voshol PJ, Fantuzzi G, Hijmans $A$ et al. The inflammasomemediated caspase-1 activation controls adipocyte differentiation and insulin sensitivity. Cell Metab 2010; 12: 593-605.

14. Vandanmagsar B, Youm YH, Ravussin A, Galgani JE, Stadler K, Mynatt RL, Ravussin E, Stephens JM, Dixit $V D$. The NLRP3 inflammasome instigates obesityinduced inflammation and insulin resistance. Nat Med 2011; 17(2): 179-188.

15. Wen $H$, Gris $D$, Lei $Y$, Jha $S$, Zhang L, Huang $M T$, Brickey WJ, Ting JP. Fatty acid-induced NLRP3-ASC inflammasome activation interferes with insulin signaling. Nat Immunol 2011; 13: 408-415.

16. Boyman O, Cho JH, Sprent J. The role of interleukin-2 in memory CD8 cell differentiation. Adv Exp Med Biol 2010; 684: 28-41.

17. Jung UJ, Choi MS. Obesity and Its Metabolic Complications: The Role of Adipokines and the Relationship between Obesity, Inflammation, Insulin Resistance, Dyslipidemia and Nonalcoholic Fatty Liver Disease. Int J Mol Sci 2014; 15(4): 6184-6223.

18. Wahid A, Hamed AN, Eltahir HM, Abouzied MM. Hepatoprotective activity of ethanolic extract of Salix subserrata against $\mathrm{CCl}_{4}$-induced chronic hepatotoxicity in rats. BMC Complement Altern Med 2016; 16(263): 110.

19. Mayra Moura F, Mariana Mader MM, Poliana MD, Marcelo HN, Bruno BB. Glycemic control and the production of cytokines in diabetic patients with chronic periodontal disease. RGO, Rev Gaúch Odontol, Porto Alegre 2015; 63 (4): 432-438.

20. Velde $A A$, Huijbens RJ, Heije $K, J E$ de V, Figdor CG. Interleukin-4 (IL-4) inhibits secretion of IL-1 beta, tumor necrosis factor alpha, and IL-6 by human monocytes. Blood 1990; 76(7): 1392-1397.

21. Bird JJ, Brown DR, Mullen AC, Moskowitz NH, Mahowald $M A$, Sider JR. Helper $T$ cell differentiation is controlled by the cell cycle. Immunity 1998; 9: 229-237.

22. Cote-Sierra J, Foucras G, Guo L, Chiodetti L, Young HA, Hu-Li J, Zhu J, Paul WE. Interleukin 2 plays a central role in Th2 differentiation. Proc Natl Acad Sci U S A 2004; 101(11): 3880-3885. 